\section{Effects of adherence to generation rules on conceptual judgments*}

\author{
CHRIS T. BERSTED ${ }^{\dagger}$ and SELBY H. EVANS \\ Texas Christian University, Fort Worth, Tex. 76129
}

Ss were given 96 trials on a simultaneous same-different similarity-dissimilarity task with histoform stimuli exhibiting different amounts of adherence to two generation rules. Similarity judgments and number of same-correct responses were found to be a positive function of amount of adherence to a generation rule. Conversely, performance was poorer (i.e., fewer correct, or higher similarity, ratings) as adherence increased on different-correct trials. It was suggested that Ss at least partially respond to learned attributes, but that these attributes are not well distinguished as defining separate classes.

Recent research in the perceptual-cognitive domain (Bersted, Brown, \& Evans, 1969; Posner \& Keele, 1968; Brown \& Evans, 1969) has dealt with Ss' increasing sensitivity to underlying prototypes or stimulus generation rules. The present research was designed to extend this line of research. Specifically, the research assessed influence of class membership upon same-different judgments and similarity ratings in a simultaneous judgment task with multidimensional stimuli.

One would predict that, as Ss learn the defining attributes of classes, pairs of stimuli drawn from the same class would result in more "same" or "highly similar" responses than pairs drawn from different classes. Moreover, given stimuli from the same class, increasing numbers of "same" responses or higher similarity ratings would be expected when the stimuli show few deviations from the probabilistic generation rule than when they show greater deviations. Conversely, given stimuli from different classes, there should be fewer "same" responses and lower similarity ratings when the stimuli adhere closely to their respective generating rules. This latter result would be expected because two stimuli exhibiting close adherence to nonoverlapping generation rules should be maximally discriminable, orse the defining attributes have been learned.

Finally, one would expect ratings of similarity and number of "same" responses to increase with practice when stimuli are drawn from the same class. This prediction

\footnotetext{
*This research was supported by the Department of Defense, Project THEMIS Contract (DAAD05-680C-0176), under the Department of Army to the Institute for the Study of Cognitive Systems through the TCU Research Foundation. Further reproduction is authorized to satisfy needs of the U.S. government.

†Present address: Department of Psychology. Southwestern Missouri State College, Springfield,
} Mo. 65802 . is based on the assumption that these responses would reflect the learning of class-defining attributes.

\section{SUBEJCTS}

Twenty Ss drawn from undergraduate psychology classes were used in this experiment.

\section{STIMULI}

The stimuli used in this investigation were produced by the VARGUS 7 (Evans, 1967) pattern generating system. Stimuli generated by this system in the form of 24 column histoforms exhibit sequential dependencies between column heights. Adherence to a generation rule for any stimulus is measured by its POSS value (proportions of transitions between column heights in common with the generation rule; Bersted, Brown, \& Evans, 1968). Stimuli from two different classes (i.e., different most-probable sequences of column heights) were selected with POSS values of $.96, .87$, and .78 , corresponding respectively to one, three, or five transitions between column heights which deviated from the generation rule. Twenty-four same-correct and 24 different-correct trials were constructed, such that all possible combinations of three POSS levels, class membership, and left-right position on a page were utilized. The pairs of stimuli thus generated were assembled into booklets characterized by two random orderings of the stimulus pairs.

Written instructions were meant to familiarize Ss with the subsequent task and to convey the notion that there can be differences within classes of objects, as well as between classes. A six-trial same-different similarity-dissimilarity task with handwritten words was used to familiarize the Ss with the subsequent task.

The Ss' task was to look at each pair of stimuli in a booklet and make both a same-different (class) judgment by circling an "S" or a "D," and then to rate the similarity of the stimuli by making a mark on a continuum. The end points of the continuum were labeled "highly similar" and "highly dissimilar." The Ss were given $15 \mathrm{sec}$ to look at each pair of stimuli and to mark their answer sheets. After completion of 48 trials, the Ss were given a second booklet, which contained the same stimulus pairs as they had just judged, but in a second random order. Half the Ss received the booklets in reverse order. No knowledge of results was given the Ss.

\section{RESULTS}

To test the hypotheses concerning similarity ratings, product moment correlations were computed between similarity judgments (11-point scale) and POSS combinations of the stimuli in each block of 48 trials. These correlations were computed for trials on which stimuli were drawn from the same class and matched in POSS values. Correlations for the two blocks of 48 trials were .62 and .67 , respectively $(\mathrm{p}<.01, \mathrm{df}=58)$. Similar correlations for different-correct trials between POSS combinations and similarity ratings were $.36(\mathrm{p}<.01, \mathrm{df}=58)$ for the first 48 trials and $.23(\mathrm{p}<.10, \mathrm{df}=58)$ for the second block of 48 trials. Figure 1 presents the mean similarity judgments made by all Ss for each block of 48 trials, with judgments on same-correct and different-correct trials plotted separately at each of the six POSS combinations.

Separate analyses of variance were computed for same-correct and different-correct trials with groups (i.e., booklet order administered first) as a between-S factor and blocks of 48 trials and POSS combinations as within-S factors. Number correct (same-different) was the dependent variable. The analysis of same-correct trials yielded a significant POS combination factor $[F(5,90)=26.99, \quad p<.01]$ and $a$ significant blocks and POSS combinations interaction $[F(5,90)=3.80, p<.01]$. No other factors or interactions were significant. The analysis of different-correct trials also yielded a significant POSS combinations factor $[F(5,90)=5.28, p<.01]$, with no other effects approaching significance. Figure 2 depicts the mean proportion of trials correct for each block of 48 trials across all Ss, with same- and different-correct trials plotted separately at each of the six POSS combinations.

\section{DISCUSSION}

The hypothesis that performance on same-correct trials would be a positive function of the POSS combinations of stimuli was supported in both the first and the second blocks of 48 trials. The interaction found between POSS combinations and blocks of trials as shown in Figs. 1 and 2 was not anticipated. It had 


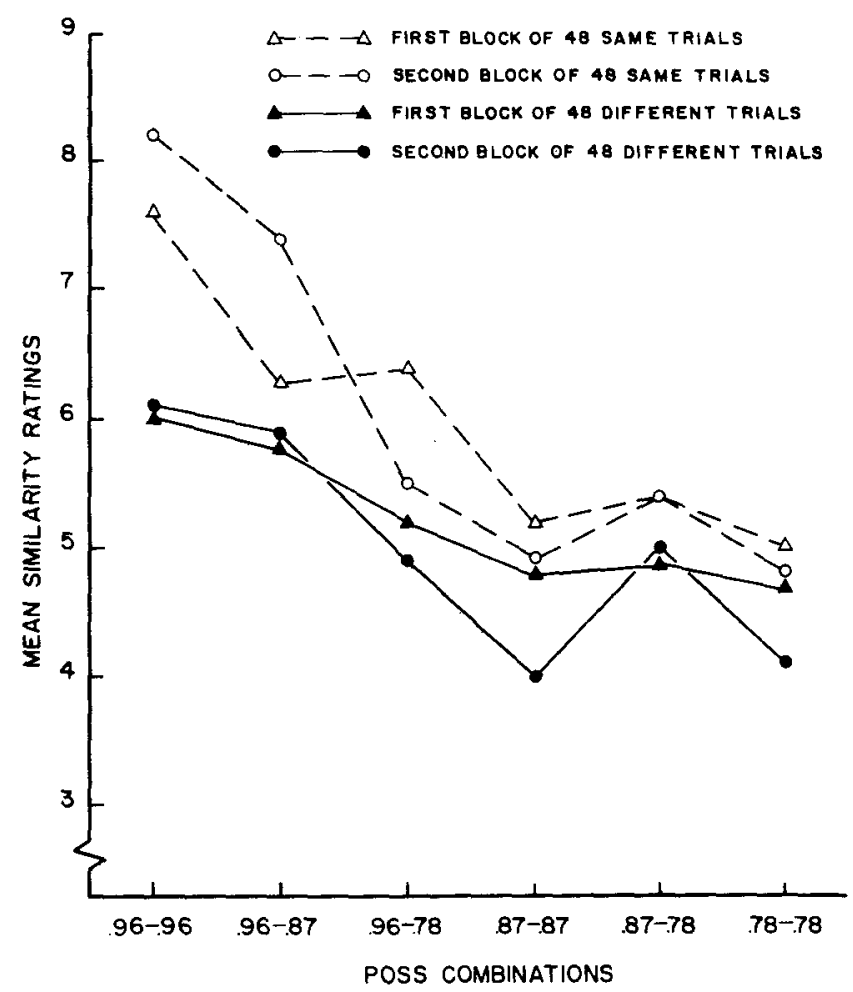

Fig. 1. Mean similarity ratings as a function of blocks of trials, POSS combinations, and type of trial.

been expected that performance (similarity ratings or number correct) would increase as a function of practice, with the implication that the increase would occur at all POSS combinations. The results, on the other hand, suggest individuals do, as a function of practice, learn to identify stimuli generated by the same rule as belonging to the same class, when they closely adhere to the generation rule. In addition, it appears that the Ss tend to adopt a conservative criterion; that is, they tend to judge stimuli as highly similar or from the same class only when they closely adhere to the generation rule.

The hypothesis that performance on different-correct trials would result in higher similarity ratings and fewer correct "different" responses at lower POSS combinations was not supported. The similarity ratings (Fig. 1) show that as pairs of stimuli adhere more closely, either to one generation rule or to each of two different generation rules, the pair is judged more similar. It should be noted, however, that judgments of "more similar" were found in every comparison of same-correct trials with different-correct trials at each of the POSS combinations. This result shows that $S$ s do to some extent

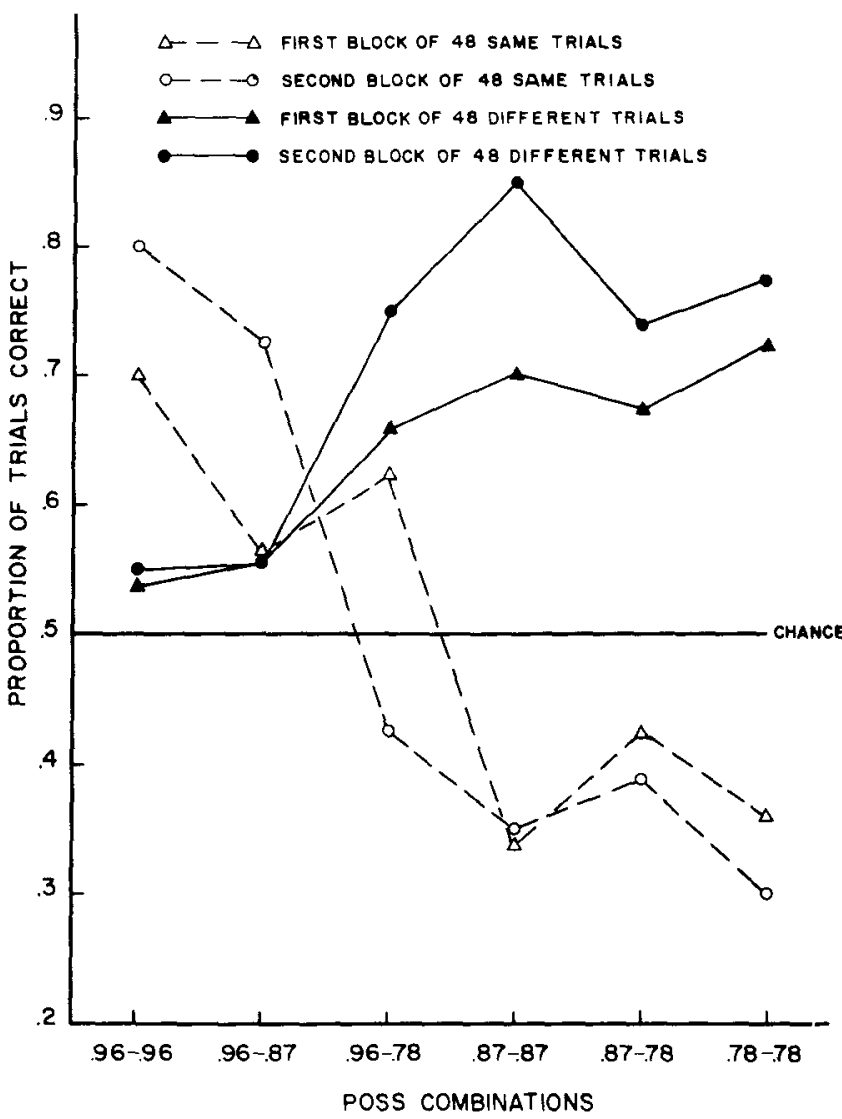

Fig. 2. Mean proportion correct as a function of blocks of trials, POSS combinations, and type of trial. discriminate trials which have two instances of the same class from those which have two instances of different classes.

The results found on different-correct trials could have two explanations. First, Ss might respond only to "regularities," or redundancy, exhibited by the stimuli. The finding that Ss do, at least to some extent, discriminate between same-correct and different-correct trials, however, makes this explanation insufficient. Second, Haygood \& Bourne's (1965) distinction between attribute and rule learning seems applicable to the present results. The Ss must learn the correct attributes of the stimuli and a rule for combining these attributes to discriminate between the classes. In the present case, attributes are defined as sequential dependencies between column heights. What may be happening is that Ss are very sensitive to the relevant attributes of the stimuli, as indicated by repetitive combinations of column heights, but that the Ss have only partially associated the attributes with each other, so as to create a basis for assignment to class. In other words, attribute learning has, to a large extent, occurred, but rule learning has occurred only to a lesser degree.
The present results suggest, as Haygood \& Bourne (1965) suggested previously, that attribute and rule learning can be independent or sequential processes. Future research should be directed toward determining whether these processes may be more nearly simultaneous, or dependent, in other tasks, as, for example, when a stringent memory requirement is added.

\section{REFERENCES}

BERSTED, C. T., BROWN, B. R., \& EVANS, S. $H$. A standard set of VARGUS 7 patterns at three levels of schematic redundancy. Psychonomic Monograph Supplements, 1968, 2(13, Whole No. 29), 251-282.

BERSTED, C. T., BROWN, B. R., \& EVANS, S. H. Free sorting with stimuli clustered in a multidimensional attribute space. Perception \& Psychophysics, 1969, 6, 409-414.

BROWN, B. R., \& EVANS, S. H. Perceptual learning in pattern discrimination tasks with two and three schema categories. Psychonomic Science, 1969, 15, 101-103.

EVANS, S. H. VARGUS 7: Computed patterns from Markov processes. Behavioral Science, $1967,12,323-328$.

HAYGOOD, R. C., \& BOURNE, L. E., JR. A trribute and rule learning aspects of conceptual behavior. Psychological Review, 1965, 72, 175-195.

POSNER, M. I., \& KEELE, S. W. On the genesis of abstract ideas. Journal of Experimental Psychology, 1968, 77, 353-363. 